\title{
Corrigendum
}

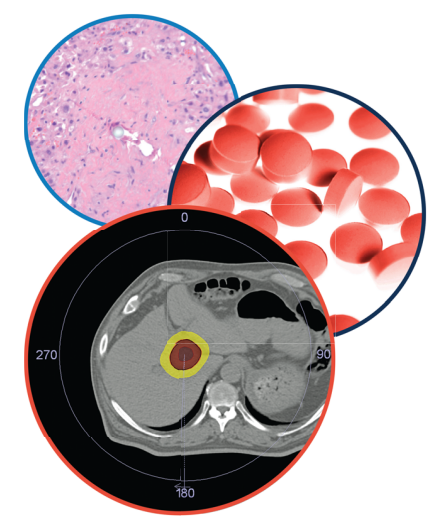

\section{Hepatic Oncology}

The Case Report by authors Roberto Iezzi, Maurizio Pompili, Eleonora Brigida Annicchiarico, Matteo Garcovich, Massimo Siciliano, Antonio Gadbarrini \& Riccardo Manfredi "Hug sign': a new radiological sign of intraprocedural success after combined treatment for hepatocellular carcinoma', which appeared in the July 2017 issue of Hepatic Oncology (4)3, 69-73 (2017), was published with the authors' names as Iezzi Roberto, Pompili Maurizio, Annicchiarico Eleonora Brigida, Garcovich Matteo, Siciliano Massimo, Gasbarrini Antonio \& Manfredi Riccardo. This has now been corrected.

The authors and editors of Hepatic Oncology would like to sincerely apologize for any inconvenience or confusion this may have caused our readers. 
